Recepción: 20 / 01 / 2017

Aceptación: 20 / 02 / 2017

Publicación: 15 / 03 / 2017

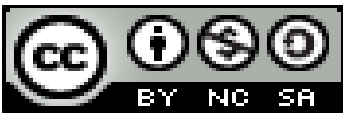

Ciencias Médicas

Caso Clínico

\title{
Aborto séptico; síndrome de Mondor: Reporte de Caso
}

\author{
Septic abortion; Mondor syndrome: Case Report
}

\section{Aborto séptico; síndrome de Mondor: Relato de Caso}

Jhonny H. Zamora-Moreira ${ }^{\mathrm{I}}$ jhonny_zamora96@hotmail.com

Gladis E. Saguay-Llangari II gladicyta.saguay@gmail.com

Johnny X. Dávila-Flores ${ }^{\text {III }}$ dr.davila@live.com

Ecuador E. Montenegro-Moran IV ecuador.montenegrom@ug.edu.ec

Correspondencia:jhonny_zamora96@hotmail.com

I. Diploma Superior en Desarrollo Local y Salud; Medico; Hospital General Dr. Liborio Panchana Sotomayor; Santa Elena, Ecuador.

II. Medico; Hospital General Dr. Liborio Panchana Sotomayor; Santa Elena, Ecuador.

III. Diplomado en Docencia Superior; Diploma Superior en Diseño Curricular por Competencias; Magister en Salud Pública; Diplomado en Docencia Superior; Obstetra. Universidad de Guayaquil, Guayaquil, Ecuador.

IV. Magister en Gerencia en Salud para el Desarrollo Local; Diploma Superior de Cuarto Nivel en Desarrollo Local y Salud; Especialista en Gerencia y Planificación Estratégica de Salud; Especialista en Ginecología y Obstetricia; Doctor en Ciencias de la Educación Especialización Administración Educativa; Doctor en Medicina y Cirugía; Profesor de Segunda Enseñanza Especialización: Químico Biológicas; Universidad de Guayaquil, Guayaquil, Ecuador. 


\section{Resumen}

Se presenta el caso de una paciente de 29 años de edad con gestación de 15 semanas que fue candidata al aborto farmacológico con misoprostol según lo protocolizado por el Servicio de Aborto del Hospital General "Dr. Liborio Panchana Sotomayor" y el método establecido para ello. Se declaró fallo de este, se decidió realizar legrado, se extrajo un feto con signos de necrobiosis, y se inició el tratamiento antibiótico de triple esquema establecido. En el transcurso de 1 h comenzó un sangramiento vaginal leve con aumento de la frecuencia cardiaca, presiones aparentemente estables más necrosis de la punta de nariz y se trasladó a la Sala de Terapia Intensiva. La paciente fallece en menos de $24 \mathrm{~h}$ con un cuadro de fallo multiorgánico. La anatomía patológica evidencia bacterias grampositivas en forma de bastones compatibles con un Clostridiumperfringes. Se concluye con el diagnóstico de síndrome de Mondor.

Palabras clave: Aborto séptico, síndrome de mondor. 
Jhonny H. Zamora-Moreira; Gladis E. Saguay-Llangari; Johnny X. Dávila-Flores; Ecuador E. Montenegro-Moran

\section{Abstract}

The case of a 29-year-old patient with 15 weeks of gestation that was a candidate for pharmacological abortion with Misoprostol, according to the protocol of the Abortion Service of "Dr. Liborio PanchanaSotomayor" General Hospital, and to the method established to this end, was presented. When this approach failed, it was decided to perform abortion and begin a regimen of triple antibiotics. A fetus with necrobiosis signs was extracted. Over the time span of hour vaginal bleeding persisted that resulted in cardiac complications and at the point of the patient's nose indicated necrosis. The patient was referred to the ICU accordingly. The patient died in less than 24 hours suffering from multiorgan failure. Pathologic anatomy evidenced the presence of cane-shaped gram-positive bacteria, compatible with Clostridium perfringes. Mondor's syndrome diagnosis was concluded.

Key words: Septic abortion; mondor's syndrome. 


\section{Resumo}

The case of a 29-year-old patient with 15 weeks of gestation who was a candidate for pharmacological abortion with Misoprostol, according to the protocol of the Abortion Service of "Dr. Liborio PanchanaSotomayor "General Hospital, and to the method established to this end, was presented. When this approach failed, it was decided to perform abortion and begin a regimen of triple antibiotics. A fetus with necrobiosis signs was extracted. Over the time span of hours vaginal bleeding persisted that resulted in cardiac complications and at the point of the patient's nose indicated necrosis. The patient was referred to the ICU accordingly. The patient died in less than 24 hours suffering from multiorgan failure. Pathologic anatomy evidenced the presence of cane-shaped gram-positive bacteria, compatible with Clostridium perfringes. Mondor's syndrome diagnosis was concluded.

Palavras chave: Aborto séptico, síndrome de mondor. 


\section{Introducción.}

El aborto séptico constituye en la actualidad un problema médico, social y económico a nivel mundial, por la gran demanda de pacientes, elevados costos y las tasas de morbilidad y mortalidad materna. La Federación Internacional de Planificación Familiar calcula que cada año ocurren 44 millones de abortos de los cuales alrededor de 15 millones son clandestinos.La morbilidad y enfermedad están en cifras muy bajas en países desarrollados donde existen buenas prácticas anticonceptivas y el aborto suele ser legal, las estadísticas reflejan 0,4 mujeres por cada 100000 abortos legales del primer trimestre del embarazo, con $21 \%$ de mortalidad, mientras que es muy alta en países subdesarrollados o en vías de desarrollo, donde suele ser ilegal o económicamente inaccesible, se producen 125 000-250 000 muertes anuales según la OMS, con el $62 \%$ de muertes por infecciones. ${ }^{2,3}$

El aborto séptico se define como un proceso infeccioso ascendente caracterizado por una endometritis, anexitis y parametris por contaminación ovular durante las maniobras abortivas o por rotura espontánea de las membranas ovulares. Constituyen factores de riesgos el embarazo avanzado, ausencia de asepsia adecuada, dificultades técnicas en la evacuación uterina o presencia no sospechada de patógenos de transmisión sexual o de gérmenes de la flora normal potencialmente de la vagina, endocérvix, genitales externos y región perineal, que estan constituido por Enterobacterias, Pseudomonas, anaerobios como Bacteroides y Clostridium. Este último desarrolla un cuadro denominado síndrome de Mondor, situación dramática que sigue a un aborto séptico, aparece dentro de 24 a 48 h después del aborto provocado o espontáneo, es extremadamente grave con alta tasa de mortalidad alrededor de $60 \%$, provocado en el $80 \%$ de los casos por Clostridiumperfrigens, es un síndrome toxémico-hemolítico que como resultado de la 
endotoxemia ocasiona hemólisis severa acompañada de trastornos de la circulación, con shock, coagulación intravascular diseminada (CID), acidosis metabólica e insuficiencia renal aguda(IRA).

\section{Caso clínico.}

Paciente DDE de 21 años de edad, con antecedentes personales de salud e historia obstétrica G3P1A1, que es candidata al aborto farmacológico con misoprostol (MSP) en el segundo trimestre (15 semanas) según los requisitos protocolizados en (Las Normas y Procedimientos de aborto farmacológico con el uso del misoprostol en el II trimestre del embarazo. Hospital General "Dr. Liborio Panchana Sotomayor", 2014) y respaldado por las Normas, Procedimientos de Ginecología y Obstetricia y Gias Practicas del Ministerio de Salud Publica

Se comienza el método con $600 \mu \mathrm{g}$, hasta completar $1200 \mu \mathrm{g}$ a las 08:00h; a las 14:00h y a las 15:00 se produce la expulsión del feto, inicio del método se realiza legrado con placenta necróticos fétida, se impone tratamiento con penicilina clindamicina y metronidazol a las dosis establecidas por vía endovenosa, se indican complementarios.

A la hora de realizado el proceder comienza con sangramiento genital oscuro levemente, con hemodinámica normal, se lleva de nuevo a Sala de Legrado, se revisa la cavidad y se encuentra sin restos, se indica $400 \mu \mathrm{g}$ de MSP rectal y oxitóxicos, se intensifica la reposición volumétrica, se avisa al banco de sangre y a terapia intensiva se traslada al salón de imagenologìa y se le realiza $\mathrm{Rx}$ estándar de tórax antes de pasar a terapia intensiva, donde se recibe una paciente taquicárdica, hipotensa, somnolienta con sangramiento genital leve.

Se reciben complementarios: Hto 10 vol\%, coagulograma: no coagula, plaquetas $65000 \mathrm{x}$ $\mathrm{mm}^{3}$ (macroplaquetas) y plasma intensamente hemolítico, con una fórmula leucocitaria de $24,2 \mathrm{x}$ 
$10^{3}$, mielocitos $0,01 \%$, juveniles $0,03 \%$, stab $0,03 \%$, segmentados $0,74 \%$, monocitos $0,02 \%$, linfocitos $0,2 \%$ y granulaciones tóxicas del $42 \%$. Con estos resultados se plantea una CID a forma hemolítica secundaria a un aborto séptico; se decide antes de llevar al salón intensificar el soporte hemodinámico, la restitución de hemoderivados y la antibioticoterapia; pasa en terapia intensiva acoplada a ventilación mecánica con tensión arterial (TA) de 100/60 mmHg y sangramiento genital escaso. Donde aparece íctero intenso y coluria, se mantiene taquipnéica, taquicárdica, hipotensa con toma progresiva del sensorio, se continúa el tratamiento intensivo cumpliendo el protocolo del shock séptico (Protocolo para el tratamiento de la sepsis severa y shock séptico. Unidad de Cuidados Intensivos del Hospital.

Aparecen evolutivamente signos de disfunción y fallo multiorgánico: CID hemolítica con necesidad de reposición intensiva de hemoderivados, hipotensión a pesar del uso de dosis supraterapeúticas, oliguria con acidosis metabólica refractaria al tratamiento, hipoxemia severa a pesar de la FIO2 al $100 \%$ con signos radiográficos de distrés respiratorio del adulto y sangramiento digestivo alto como evento final. La paciente fallece en menos de $24 \mathrm{~h}$ de realizarse la evacuación del útero por legrado instrumental luego del aborto farmacológico.

\section{Informe anatomopatológico}

CDM: shock séptico.

CIM: sepsis generalizada por endomiometritis aguda severa con extensas áreas de absceso. CBM: aborto farmacológico. 


\section{Órganos genitales}

Macroscópico: toda la pieza incluyendo ambos anejos de color azul violáceo; serosa con adherencias fibrosas a predominio del istmo y segmento. A la apertura de la cavidad endometrial está dilatada, algo irregular y hemorrágica.

Microscópico: útero grávido con endomiometritis aguda severa con extensas áreas de absceso, necrosis hemorrágica y abundantes colonias bacterianas grampositivas (coloración especial Brown Breen positiva) que involucra cuello uterino.

\section{Comentarios.}

En este caso se está en presencia de la forma mas grave del aborto sépticosegún la clasificacion de este en aborto infectado simple o febril, complicado, séptico y síndrome de Mondor, que tiene una tríada sintomática característica compuesta por anemia, ictericia y cianosis, lo que constituye el llamado síndrome tricolor (pálido por la anemia, amarillo por la ictericia y azul por la cianosis), también llamado gangrena uterina por provocar mionecrosis del útero.Esta gravedad está dada por el corto período de incubación del Clostridiumperfringens $(24-48$ h) y la presencia de un síndrome tóxico- hemolítico que conduce a un síndrome de disfunción o fallo multiorgánico (SMO) asociado por sí solo con una alta mortalidad independiente de lo precoz y correcto del tratamiento impuesto. Esta paciente desarrolló ambas características distintivas del síndrome.

Este síndrome es causado en la mayoría de los casos por el Clostridium perfringens, aunque lo pueden originar otros gérmenes anaerobios, grampositivos y gramnegativos, bacteriológicamente pertenece al género de bacilos grampositivos, que crecen en anaerobiosis y en el caso del C. perfringens no produce esporas en los tejidos. Es el responsable de tres síndromes 
Jhonny H. Zamora-Moreira; Gladis E. Saguay-Llangari; Johnny X. Dávila-Flores; Ecuador E. Montenegro-Moran

histiotóxicosclostridiales, es el clostridio más fácilmente hallado incluyendo hemocultivos y los cultivos de sitios infectados: infecciones intraabdominales, del aparato genital femenino (se aísla en el 5-10\% de la flora genital normal) y de partes blandas.

En el caso de la paciente el germen fue observado en la muestra anatomo-patológica del útero con coloración de rutina de hematoxilina eosina y además como coloración especial Brown Brenn. A diferencia de las infecciones causadas por Staphylococcus aureus hay típicamente una ausencia de leucocitos polimorfonucleares en el sitio de infección, probablemente por la presencia de las toxinas clostridiales. Las esporas son raras en los frotis de tejido, son mas frecuentes en las coloraciones a partir de los cultivos bacterianos, ambas características distintivas del Clostridiumperfringens pueden ser observadas en la figura.

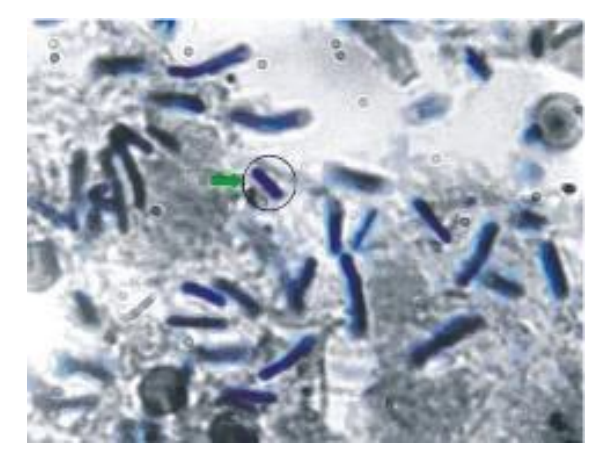

Fig. Estudio histológico.

El C. perfringens es el más frecuentemente involucrado en la gangrena gaseosa, la frecuencia llega al $80 \%$. Epidemiológicamente contribuyen al desarrollo de gangrena gaseosa la presencia de determinados factores del huésped como la rotura de las barreras cutáneo-mucosas por cirugía o traumatismo, presencia de enfermedades graves con reducida tensión de oxígeno en los tejidos, 
presencia de cuerpos extraños, insuficiencia circulatoria, tratamiento con múltiples antibióticos o tratamiento con inmunosupresores y en general, constituye parte de una infección polimicrobiana.

Se han descrito 12 toxinas producidas por este clostridio, cuatro de ellas son letales y sirven para clasificarlos en 5 especies (de la A a E) Las toxinas alfa y tetha son las involucradas en la patogénesis que conllevan a hemólisis, daño plaquetario y capilaritis diseminada con inducción de los mediadores inflamatorios causantes del estado de shock.

Se describe en su patogénesis el trauma inicial de los tejidos asociado con un pobre aporte de oxígeno, este último origina una disminución del potencial de óxido-reducción que facilita el crecimiento de los gérmenes anaerobios entre ellos el clostridio, que libera toxinas al tejido circundante con más daño tisular y paso a través de la circulación local a la sistémica con manifestaciones de toxemia. A causa del daño capilar hay aumento de la permeabilidad con edema tisular resultante, que disminuye el retorno venoso y empeora la perfusión tisular lo cual origina isquemia, hipoxia secundaria y muerte celular, de manera que se recluta más tejido dañado que favorece el crecimiento del clostridio con liberación de más toxinas dentro del tejido local y la circulación sistémica que perpetúa el cuadro de reacción inflamatoria sistémica.

El hecho de encontrarse un feto muerto con signos de necrosis en una paciente asintomática y asignológica hasta ese momento, lleva a pensar que esto fue un evento con poco tiempo de ocurrido y localizado, sin signos todavía de respuesta inflamatoria sistémica, que pudo haber sido empeorado por la isquemia tisular e hipoxia resultantes del efecto uterotónico del misoprostol en dosis repetidas y elevadas (1 $200 \mu \mathrm{g}$ ), y que se generalizó por el propio legrado uterino al romper la barrera mucosa-uterina y permitir el paso del germen y sus mediadores inflamatorios al torrente vascular, con el desarrollo a partir de ese momento del síndrome de respuesta inflamatoria sistémica 
Jhonny H. Zamora-Moreira; Gladis E. Saguay-Llangari; Johnny X. Dávila-Flores; Ecuador E. Montenegro-Moran

(SRIS) que se caracterizó inicialmente por una CID hemolítica, traducción del daño endotelial severo con activación de los mecanismos de la coagulación; posteriormente, desarrolló de forma galopante el SMO con shock severo asociado con hemólisis masiva y fallo renal como causa final de muerte como se describe clásicamente. ${ }^{4,6}$

En relación con el tratamiento este debe tener un enfoque quirúrgico- obstétrico como se realizó en este caso, ya que el tratamiento antibiótico por sí solo no es suficiente para dominar el cuadro, en esta paciente se realizó el legrado uterino cuando estaba indicado y en una paciente no clasificada hasta ese momento de alto riesgo de infección, son consideradas así a las enfermas con un cuadro febril mayor a $39{ }^{\circ} \mathrm{C}$, antecedentes de maniobras abortivas, infección que se extiende más allá del útero, tamaño uterino superior a 12 semanas, infección por $C$. perfringens sospechada por los signos clásicos de ictericia, hiperestesia cutánea y mialgias, descarga purulenta por orificio cervical externo, signos de shock (hipotensión, oliguria, disociación pulso/temperatura

Ninguna de estas condiciones se daban en esta paciente, después de realizado el legrado se diagnostica un aborto diferido infectado en una paciente ahora de alto riesgo, por encontrarse un feto necrosado que se infiere sea de origen infeccioso y se impone el tratamiento antibiótico normado para estos casos teniendo en cuenta que es siempre polimicrobiana.

Se presenta la forma clínica más grave del aborto complicado por una infección, el síndrome de Mondor, originado por un germen oportunista, agresivo y de distribución frecuente en la naturaleza. Considerando la legalidad del aborto en nuestro país que implica una alta morbilidad de este, no se debe olvidar que a pesar de ser un aborto institucional sigue siendo la infección posaborto la principal causa de morbilidad-mortalidad de este, por lo que todos los médicos deben estar preparados para el diagnóstico y tratamiento oportuno de este síndrome y recordar que ante todo 
aborto diferido con cualquier signo de sospecha de infección por clostridio y más aún que se asocie con una CID hemolítica, debe inmediatamente realizarse una histerectomía obstétrica independiente del estado de la paciente, porque es la forma más efectiva de combatir la gangrena uterina y disminuir su alta mortalidad.

Fig. Estudio histológico con lente de inmersión. Obsérvese estructura bacteriana en forma de bacilos grampositivos, largos, gruesos, de bordes romos, que por el cuadro clínico debe corresponder al género Clostridium, no hay presencia de esporas ni de leucocitos polimorfonucleares en el tejido..

\section{Agradecimiento}

Dr. Jorge MerchánVillamar, responsable del departamento de ginecología y obstetricia.

\section{Bibliografía.}

1. Phillip G, Stubblefield and Grimes DA. Septic abortion. Rev Artic. 1994 Aug; 331(5):310-4.

2. Pérez Trallero E, Cueto M, Miranda C. Bacilos grampositivos. En: de la Rosa M, Prieto J (eds). Microbiología en ciencias de la salud. Conceptos y aplicaciones. $2^{\mathrm{a}}$ ed. Madrid: Elsevier; 2003. p. 8490

3. Solari LA, Quintana BE, Yañez AA. Aborto séptico. Revista Smiba. (Fecha de acceso 12 de marzo del 2005). URL disponible en: http://www.dewebsa.com.ar./smiba/revista/smiba.htm

4. Mena C, Gutiérrez JI, Múnera W. Comportamiento del aborto séptico en pacientes que consultaron al Hospital San Vicente de Paul. Universidad de Antioquia 1994-1997. RevColombObstetGinecol. 1999 $\mathrm{Jul} / \mathrm{Sept} ; 50(2)$.

5. Dellinger RP, Carlet JM, Masur H, Gerlach H, Calandra T; Cohen J, et al. For the Surviving Sepsis Campaign.Mangement Guidelines Committee: Surviving Sepsis Campaing Guidelines for management of Severe Sepsis and Septic Shock. CritCareMed. 2004;32(3):856-73.

6. Stubblefield PG, Grimes DA. Septic Abortion. N Eng J Med. 1994:331(5).

7. Ledger WJ: Infecciones de la pelvis femenina. En: Mandell/Douglas/Bennett (eds). Enfermedades infecciosas. Principios y práctica. 3ra ed. Cap 96. New York: Editorial Médica Panamericana;1991. p. $1016-22$ 
8. Solari LA. Sepsis por aborto. Análisis de los tipos del mismo. Las fallas de órganos y su mortalidad. La Prensa Médica Argentina. 1995;82:740-3

9. Allen SD, Emery CL, lyerly DM. Clostridium . In: Murray PR, Baron EJ, Jorgensen JH, Pfaller MA, Yolken RH (eds). Manual of Clinical Microbiology. $8^{\mathrm{a}}$ ed. Washington DC : ASM Press; 2003. p. 835-56.

10. Mangels JI. Anaerobic bacteriology. In: Isenberg HD (ed). Clinical Microbiology Procedures Handbook. 2nd ed. Washington DC : ASM Press; 2004.

11. Blanco GM, Fraile MR, Domingo AA, Calvo CJ, Sastre JL, Armengol ED. Microbiología de la infección perinatal. (Fecha de acceso 5 de enero del2005). URL disponible en: http://www.seimc.org/protocolos/microbiologia/cap13.htm

12. García JE, García MI, Merino ML. Infecciones por Clostridios. Situación actual. En: García Rodríguez JA (ed). Infecciones por anaerobios 100 años después. Madrid: Sociedad Española de Quimioterapia;1994. p. 35-49.

13. Bartlett JG. Gangrena gaseosa (otras enfermedades asociadas con clostridios). En: Enfermedades infecciosas. Principios y práctica. Mandell/Douglas/Bennett (eds). 3ra ed. Cap 222. New York: Editorial Médica Panamericana; 1991. p. 1957-68.

14. Rood JI, Cole ST. Molecular genetics and pathogenesis of Clostridium perfringens. Microbiol Rev. 1991;55:621 